\title{
Haftungsfragen im Rahmen der Teleradiologie
}

Im Zeitalter der Digitalisierung ermöglicht die Telemedizin neue Formen der ärztlichen Zusammenarbeit und Patientenversorgung. Effizientere Nutzung von Expertenwissen, Vermeiung von Doppeluntersuchungen, Ausweitung der Befundmöglichkeiten sowie insgesamt eine schnellere Versorgung insbesondere in ländlichen Regionen sind hier nur einige der zu nennenden Vorteile. Insbesondere die Teleradiologie hat sich hier bereits seit längerem bewährt, sodass es längst kein Einzelfall mehr ist, wenn der Radiologe nicht vor Ort, sondern aus der benachbarten Klinik Röntgenbilder befundet. Im Zuge des technischen Fortschritts entstanden in den vergangenen Jahren verschiedene Projekte zur Weiterentwicklung der Teleradiologie, die aber auch rechtliche Fragen aufgeworfen haben, insbesondere im Hinblick auf die jeweiligen Verantwortungsbereiche. Um Sicherheit für alle Beteiligten zu schaffen, sollen daher die Grundzüge der Haftung bei der Nutzung der Teleradiologie dargestellt werden.

\section{Haftung je nach Beteiligungsgrad $\nabla$}

Bei der praktischen Ausgestaltung der Teleradiologie bieten sich vielfältige Gestaltungsmöglichkeiten, bei denen der extern hinzugezogene Radiologe je nach Konstellation mehr oder weniger Einfluss auf die diagnostischen oder therapeutischen Prozesse hat. Das Spektrum reicht hier von geringem bzw. kaum nachweisbarem Einfluss auf die weitere Versorgung der Patienten bis hin zur Übernahme der „Herrschaft des Behandlungsgeschehens“ durch den Spezialisten. Für die Frage der Haftung ist entscheidend, inwieweit der hinzugezogene Radiologe am Behandlungsgeschehen beteiligt ist und ob sein Beitrag als (Mit-) Behandlung zu qualifizieren ist, da er in diesem Fall auch für eigene Fehler gegenüber dem Patienten haftet. Für die Qualifizierung als Mitbehandlung ist dabei grundsätzlich unbeachtlich, dass der Patient in aller Regel mit dem hinzugezogenen Radiologen nicht in vertraglichen Beziehungen steht.

\section{Telediagnostik nach § 3 Abs. 4 RöV}

Bei der Telediagnostik nach $\S 3$ Abs. 4 der Röntgenverordnung wird die Indikationsstellung und Befundung vollständig vom ortsabwesenden Radiologen vorgenommen, etwa an Kliniken außerhalb der Dienstzeiten sowie in unterversorgten Gebieten. Hier ist die Frage der Haftung einfach zu beantworten, da die Verantwortung allein beim hinzugezogenen Radiologen liegt mit der Folge, dass dieser für Fehler gegenüber dem Patienten unmittelbar haftet. Eine Haftung des vor Ort anwesenden Arztes für Fehler des Radiologen besteht dagegen grundsätzlich nicht.

\section{Fachgleiches und fachfremdes \\ Telekonsil}

Schwieriger ist die Haftungsfrage schon beim sogenannten Telekonsil zu beantworten, da hierunter verschiedene Konstellationen fallen können. Die Bezeichnung als Telekonsil ist dabei für die Haftung in erster Linie nicht entscheidend, sodass in jedem Einzelfall zu untersuchen ist, inwiefern der hinzugezogene Radiologe die Behandlung tatsächlich beeinflusst. Beim Telekonsil ist zunächst zu unterscheiden, ob es zwischen Fachärzten des gleichen Fachgebietes oder Fachärzten unterschiedlicher Fachgebiete stattfindet: Zieht der behandelnde Radiologe einen externen Radiologen, etwa aufgrund von dessen Expertenwissen, telekonsiliarisch zu Rate, um sich gemeinsam nach vorangegangener Untersuchung zu beraten und eine Diagnose zu stellen bzw. Therapie festzulegen, liegt die Verantwortung für die Behandlung und damit die Haftung in der Regel allein bei den vor Ort behandelnden Radiologen. Die Mitwirkung des hinzugezogenen Experten beschränkt sich in diesen Fällen zumeist auf die Beratungsleistung im Verhältnis zum behandelnden Arzt, die keinen unmittelbaren Einfluss auf die Behandlung ausübt, sodass kein Behandlungsverhältnis zwischen hinzugezogenem Radiologen und Patient entsteht. Denkbar sind allerdings auch hier Fälle, in denen der hinzugezogene Experte aufgrund seines überlegenen Wissens die Behandlung derart steuert und in den Behandlungsplan eingreift, dass sein Beitrag als Mitbehandlung zu qualifizieren ist. In diesen Fällen kommt neben der Haftung des behandelnden Radiologen auch eine Haftung des hinzugezogenen Radiologen in Betracht.

Im Fall des fachfremden Telekonsils wendet sich ein Arzt eines anderen Fachgebiets an den Radiologen, um seine eigene fehlende Fachkompetenz zu überbrücken. Hier handelt es sich faktisch um einen Fall der Mitbehandlung, da der hinzugezogene Radiologe durch die verbindliche Diagnosestellung und Festlegung der weiteren Behandlung maßgeblich das Behandlungsgeschehen beeinflusst und daher für Fehler in diesem Zusammenhang die volle Verantwortung trägt. Solange hier keine offensichtlichen Fehler des Radiologen vorliegen, kann sich der behandelnde fachfremde Arzt vor Ort grundsätzlich auf die Meinung des Experten verlassen und ist mangels Verschuldens nicht haftbar.

\section{Haftung beim Einholen einer Zweitmeinung}

Ähnlich wie beim Telekonsil unter Radiologen verhält es sich mit der Haftung bei Einholen einer 2. Meinung, wenn der behandelnde Arzt einen Radiologen zu Rate zieht, um die von ihm gestellte Diagnose lediglich überprüfen bzw. bestätigen zu lassen. Hier greift der hinzugezogene Radiologe regelmäßig nicht lenkend in das Behandlungsgeschehen ein, es findet lediglich ein kollegialer Austausch beruhend auf einer bereits bestehenden Tendenz statt. Aufgrund des Ergebnisses der Beratung trifft der behandelnde Arzt vor Ort dann hinsichtlich der Weiterbehandlung eine eigenverantwortliche Entscheidung, für die er voll haftet. Denkbar ist jedoch auch hier im Einzelfall, dass der behandelnde Arzt allein aufgrund der anderslautenden Meinung des hinzugezogenen Radiologen seine Diagnose oder den Behandlungsplan ändert. In diesen Fällen kommt daneben auch eine Haftung des radiologischen Beraters in Betracht.

\section{Haftung für technische Fehler $\nabla$}

Problematisch ist die Haftungsverteilung, wenn zwar der Rat des hinzugezogenen Radiologen aufgrund der ihm vorgelegten Daten richtig ist, die Daten aber ihrerseits fehlerbehaftet sind. $\mathrm{Zu}$ denken ist beispielsweise an die Übermittlung falscher 
Bilder nach Patientenverwechslung oder an eine schlechte Bildqualität. Liegt die fehlerhafte Übermittlung in der Sphäre des vor Ort behandelnden Arzts, trägt er die Verantwortung für eine falsche Diagnose. Der beratende Radiologe haftet in diesen Fällen nur dann, wenn die fehlerhafte Übertragung für ihn offensichtlich ist und er es unterlassen hat, die Daten einer gewissen Plausibilitätskontrolle zu unterziehen.

\section{Fazit}

\section{$\nabla$}

Pauschale Aussagen über die jeweilige Haftung im Anwendungsbereich der Teleradiologie lassen sich gerade im Zusammenhang mit telekonsiliarischen Beratungen nicht treffen. Entscheidend für die Frage der Haftung ist daher stets im konkreten Einzelfall, wer das eigentliche Behandlungsgeschehen beherrscht. Beschränkt sich die Tätigkeit des zugezogenen Radiologen auf reine Beratungsleistungen, haftet nur der behandelnde Arzt. Prägt er dagegen die weitere Behandlung durch sein Spezialwissen, ist er für Diagnosefehler verantwortlich, wenn sich der behandelnde Arzt vor Ort auf seine Fachkompetenz verlassen konnte. Tragen sowohl der behandelnde Arzt als auch der hinzugezogene Radiologe arbeitsteilig zur weiteren Behandlung bei, haben hingegen beide dafür Sorge zu tragen, dass diese fehlerfrei erfolgt. Um die jeweiligen Verantwortungsbereiche sicher voneinander abzugrenzen, empfiehlt sich insbesondere für den vor Ort behandelnden Arzt eine sorgfältige Dokumentation und Begründung der eigenen Therapieentscheidung. Grundsätzlich gilt, dass der Patient durch die Nutzung der Teleradiologie nicht schlechter gestellt sein darf als bei einer Befundung vor Ort. Zur Vermeidung von Haftungsrisiken sollte beim Einsatz von Telemedizin daneben besondere Aufmerksamkeit auf die Aufklärung des Patienten gelegt werden.

Rechtsanwalt Dr. A. Wienke, Fachanwalt für Medizinrecht

Rechtsanwältin R. Sailer, LL.M. Fachanwältin für Medizinrecht

Wienke E Becker - Köln

Mail: AWienke@Kanzlei-WBK.de 